European Physical Journal B 73, 483-487 (2010)

\title{
Microscopic Bardeen-Cooper-Schrieffer formulation of the critical temperature of multilayer copper-oxide superconductors
}

\author{
George Kastrinakis* \\ Institute of Electronic Structure and Laser (IESL), \\ Foundation for Research and Technology - Hellas (FORTH), P.O. Box 1527, Iraklio, Crete 71110, Greece
}

(Dated: Received 22 October 2009 / In final form 8 December 2009)

\begin{abstract}
We study superconductivity in multilayer copper oxides, in the frame of a realistic microscopic formulation. Solving the full temperature dependent BCS gap equations, we obtain a maximum in the transition temperature $T_{c}$ for $\mathrm{M}=3$ or $4 \mathrm{CuO}_{2}$ layers in the unit cell for appropriate values of the interlayer tunneling (negative pair tunneling), and via the consideration of the doping imbalance between the inner and outer layers. This is the ubiquitous experimental result for Ca intercalated copper oxides, as opposed to other intercalating elements. Further, using a restricted set of parameters, we obtain an exact fit of $T_{c}(\mathrm{M}=1-4)$ for five different Ca intercalated homologuous copper oxide families.
\end{abstract}

PACS numbers: 74.72.-h, 74.20.Fg, 74.62.-c, 74.78.Fk

A long standing puzzle of the high transition temperature $T_{c}$ cuprate superconductors is the dependence of $T_{c}$ on the number $\mathrm{M}$ of $\mathrm{CuO}_{2}$ layers in the unit cell. Experiments show ubiquously that within any Ca intercalated homologuous cuprate family, i.e. for materials sharing the same charge reservoir block but having varying $\mathrm{M}$ (here $\mathrm{Ca}$ is between the layers), $T_{c}$ is maximum for $\mathrm{M}=3$ - a summary of experiments appears in e.g. [1] - with the $\mathrm{M}=4$ exception of the $\mathrm{Tl}_{2} \mathrm{Ba}_{2} \mathrm{Ca}_{M-1} \mathrm{Cu}_{M} \mathrm{O}_{2 M+3+\delta}$ family [2], following a dome-type dependence on $\mathrm{M}$. That is at optimum doping for any M. As mentioned in [3], other spacing elements, such as Y, Ba and Sr yield lower $T_{c}$ for the multilayer materials compared to the single layer material. Setting aside the central issue of superconductivity within a single $\mathrm{CuO}_{2}$ layer, the issue of what determines $T_{c}$ in a multilayer system is a very important one. Inter alia, it points to the role of out-of-plane mechanisms and to how $T_{c}$ can be maximized for any given cuprate family.

This issue has been treated in a number of theoretical papers, as in 1, 4 [10], with limited success. E.g. in ref. 1] a Bardeen-Cooper-Schrieffer (BCS) model (different from ours) was considered, and a saturating increase of $T_{c}$ as a function of $M=1-\infty$ was obtained. In ref. [10] the dome with a maximum for $M=3$ was obtained for the superconducting order parameter (not directly for $T_{c}$ itself) in the frame of a Landau-Ginzburg functional treatment, and by appealing to the putative ddw order parameter.

The cuprates fall into the realm of strong correlations, thus calling for an Eliashberg-type treatment 11 13. Given the lack of a definitive theory for the single $\mathrm{CuO}_{2}$ layer superconducting state, our BCS treatment is a decent attempt towards the understanding of the multilayer cuprates. We hereby consider a realistic microscopic model in the frame of the BCS formulation [14], which does yield the experimental fact of the maximum $T_{c}$ for $\mathrm{M}=3$ or 4 for a range of the parameters considered. That is for moderately small single particle and pair tunneling between successive $\mathrm{CuO}_{2}$ layers. As experiments indicate that $T_{c}$ decreases steadily for $\mathrm{M}>3$ (with the aforementioned exception), we content ourselves with calculating $T_{c}$ for the cases $\mathrm{M}=1-4$, assuming that the trends found also apply for $\mathrm{M}>4$. The complex calculations required for $\mathrm{M}>4$ are out of the scope of the present paper.

We consider the BCS-type Hamiltonian for $\mathrm{M} \mathrm{CuO}_{2}$ layers in the unit cell

$$
\begin{aligned}
& H=\sum_{i ; k, \sigma} \varepsilon_{i, k} d_{i, k, \sigma}^{\dagger} d_{i, k, \sigma}+\sum_{i ; k, p} V_{i}(k, p) d_{i, k, \uparrow}^{\dagger} d_{i,-k, \downarrow}^{\dagger} d_{i,-p, \downarrow} d_{i, p, \uparrow} \\
& +\sum_{<i l>, k, \sigma} t_{\perp, k} d_{i, k, \sigma}^{\dagger} d_{l, k, \sigma}+\sum_{<i l>, k} T_{k} d_{i, k, \uparrow}^{\dagger} d_{i,-k, \downarrow}^{\dagger} d_{l,-k, \downarrow} d_{l, k, \uparrow} .
\end{aligned}
$$

The creation/annihilation operators $d_{i, k, \sigma}^{\dagger} / d_{i, k, \sigma}$ describe electrons in the $i$-th $\mathrm{CuO}_{2}$ layer in the unit cell, interacting via $V_{i}(k, p)$, and $i=1-\mathrm{M} . \varepsilon_{i, k}=\epsilon_{i, k}-\mu_{i}$, with the dispersion $\epsilon_{i, k}=-2 t_{i}\left(\cos k_{x}+\cos k_{y}\right)-4 t_{i}^{\prime} \cos k_{x} \cos k_{y}-2 t_{i}^{\prime \prime}\left(\cos 2 k_{x}+\right.$ $\left.\cos 2 k_{y}\right), k_{x}, k_{y}=[-\pi, \pi]$, and the chemical potential $\mu_{i}$ of the $i$-th layer. The (in-plane momentum conserving) coupling between successive neighbor $\mathrm{CuO}_{2}$ layers $\langle i l\rangle$ for single electron tunneling is [15]

$$
t_{\perp, k}=t_{o}\left(\cos k_{x}-\cos k_{y}\right)^{2},
$$

while for pair tunneling is (proportional to $t_{\perp, k}^{2}$, e.g. c.f. [6] and below)

$$
T_{k}=T_{o}\left(\cos k_{x}-\cos k_{y}\right)^{4} .
$$


We diagonalize the single particle kinetic energy part of the Hamiltonian. The original operators $d_{i, k, \sigma}$ are given in terms of the new ones $c_{i, k, \sigma}$ as $\vec{d}_{M}=U_{M} \vec{c}_{M}, \vec{d}_{M}=\left(d_{1, k, \sigma}, d_{2, k, \sigma}, \ldots, d_{M, k, \sigma}\right)$ [16]. In the new basis, we consider variational BCS states of the type

$$
\left|\Psi>=\prod_{i, k}\left(u_{i, k}+v_{i, k} c_{i, k, \uparrow}^{\dagger} c_{i,-k, \downarrow}^{\dagger}\right)\right| 0>
$$

with $u_{i, k}, v_{i, k}$ the well known coherence factors.

For $\mathrm{M}=2$ layers $c_{1,2}=\left(d_{1} \pm d_{2}\right) / \sqrt{2}$, and the transformed Hamiltonian is

$$
\begin{aligned}
H=\sum_{i ; k, \sigma} \xi_{i, k} c_{i, k, \sigma}^{\dagger} c_{i, k, \sigma}+ & \frac{1}{2} \sum_{k, p} V(k, p)\left[c_{1, k, \uparrow}^{\dagger} c_{1,-k, \downarrow}^{\dagger} c_{1,-p, \downarrow} c_{1, p, \uparrow}+c_{1, k, \uparrow}^{\dagger} c_{1,-k, \downarrow}^{\dagger} c_{2,-p, \downarrow} c_{2, p, \uparrow}+(1 \leftrightarrow 2)\right] \\
& +\frac{1}{2} \sum_{k} T_{k}\left[c_{1, k, \uparrow}^{\dagger} c_{1,-k, \downarrow}^{\dagger} c_{1,-k, \downarrow} c_{1, k, \uparrow}+c_{1, k, \uparrow}^{\dagger} c_{1,-k, \downarrow}^{\dagger} c_{2,-k, \downarrow} c_{2, k, \uparrow}+(1 \leftrightarrow 2)\right] .
\end{aligned}
$$

Here $\xi_{1, k}=\varepsilon_{k}-t_{\perp, k}, \xi_{2, k}=\varepsilon_{k}+t_{\perp, k}$ correspond to the bonding and antibonding states. The two initial layers are equivalent, and hence $V_{1}=V_{2}=V$. We also made use of the fact that the only non-zero matrix elements with four operators $c_{i, k, \sigma}(<O>=<\Psi|O| \Psi>)$ are - c.f. ref. [14] and below for $u_{i, k}, v_{i, k}$ and $f_{i, k}$

$$
<c_{i, k, \uparrow}^{\dagger} c_{i,-k, \downarrow}^{\dagger} c_{j,-p, \downarrow} c_{j, p, \uparrow}>=v_{i, k} u_{i, k} v_{j, p} u_{j, p}\left(1-2 f_{i, k}\right)\left(1-2 f_{j, p}\right)
$$

Bearing in mind that $u_{i, k}^{2}+v_{i, k}^{2}=1, \quad 0 \leq u_{i, k}^{2}, v_{i, k}^{2} \leq 1$, we treat the coherence factors as $u_{i, k}=\cos \left(\theta_{i, k}\right)$, $v_{i, k}=\sin \left(\theta_{i, k}\right)$. Minimizing $<H>=<\Psi|H| \Psi>$ with respect to $\theta_{i, k}$ yields the gap equations

$$
0=-2 \xi_{i, k} v_{i, k} u_{i, k}+\Delta_{i, k}\left[u_{i, k}^{2}-v_{i, k}^{2}\right]
$$

while minimizing $<F=H-T S>$, with the entropy $S=-2 \sum_{i, k}\left[f_{i, k} \ln f_{i, k}+\left(1-f_{i, k}\right) \ln \left(1-f_{i, k}\right)\right]$ and the temperature $T=1 / \beta$, with respect to the thermal factors $f_{i, k}$ yields

$$
f_{i, k}=\frac{1}{1+e^{\beta E_{i, k}}} \quad, \quad E_{i, k}=\frac{\xi_{i, k}}{u_{i, k}^{2}-v_{i, k}^{2}} .
$$

The gaps $\Delta_{i, k}$ are a sum of a diagonal (in the layer index $i$ ) part $G_{i, k}$ and a non-diagonal part $g_{i, k}$.

$$
\begin{gathered}
\Delta_{i, k}=G_{i, k}+g_{i, k}, G_{i, k}=-\frac{1}{2} \sum_{p} V(k, p) v_{i, p} u_{i, p} \tanh \left(\beta E_{i, p} / 2\right)-\frac{1}{2} T_{k} v_{i, k} u_{i, k} \tanh \left(\beta E_{i, k} / 2\right), \\
g_{i, k}=-\frac{1}{2} \sum_{p} V(k, p) v_{j, p} u_{j, p} \tanh \left(\beta E_{j, p} / 2\right)-\frac{1}{2} T_{k} v_{j, k} u_{j, k} \tanh \left(\beta E_{j, k} / 2\right), \quad(i, j)=\{(1,2),(2,1)\} .
\end{gathered}
$$

Here $\Delta_{1, k}=\Delta_{2, k}$. We note that setting $i=j$ and $T_{k}=t_{\perp, k}=0$ reduces these equations to the usual gap equation $0=-2 \xi_{k} v_{k} u_{k}+\Delta_{k}\left[u_{k}^{2}-v_{k}^{2}\right]$, with $\Delta_{k}=-\sum_{p} V(k, p) v_{p} u_{p} \tanh \left(\beta E_{p} / 2\right)$ and $E_{k}=\sqrt{\xi_{k}^{2}+\Delta_{k}^{2}}=\xi_{k} /\left(u_{k}^{2}-v_{k}^{2}\right)$. But, note that we do not enforce a relation of the type $E_{i, k} \sim \sqrt{\Delta_{i, k}^{2}+\xi_{i, k}^{2}}$ for $\mathrm{M}>1$.

The general form of the transformed Hamiltonian is

$$
H=\sum_{i, k, \sigma} \xi_{i, k} c_{i, k, \sigma}^{\dagger} c_{i, k, \sigma}+\sum_{i, j ; k, p} w_{i j}(k, p) c_{i, k, \uparrow}^{\dagger} c_{i,-k, \downarrow}^{\dagger} c_{j,-p, \downarrow} c_{j, p, \uparrow}
$$

The coefficients $w_{j i}(k, p)$ are determined below. Thus we obtain as above the gap equations for $u_{i, k}, v_{i, k}, f_{i, k}$ in the general form (7), (8), but now with

$$
G_{i, k}=-\sum_{p} w_{i i}(k, p) v_{i, p} u_{i, p} \tanh \left(\beta E_{i, p} / 2\right), g_{i, k}=-\frac{1}{2} \sum_{j \neq i ; p}\left[w_{i j}(k, p)+w_{j i}(k, p)\right] v_{j, p} u_{j, p} \tanh \left(\beta E_{j, p} / 2\right) .
$$

For $\mathrm{M}=3$ layers, $\xi_{1, k}=\varepsilon_{1, k}, \xi_{(2,3), k}=\left(\varepsilon_{1, k}+\varepsilon_{2, k} \mp d\right) / 2$, with $d=\sqrt{a^{2}+8 t_{\perp, k}^{2}}, a=\varepsilon_{1, k}-\varepsilon_{2, k}$. Also

$$
w_{i j}(k, p)=V_{1}(k, p)\left[A_{i j}^{(1)}(k, p)+A_{i j}^{(3)}(k, p)\right]+V_{2}(k, p) A_{i j}^{(2)}(k, p)+E_{i j}(k) \delta_{k p}
$$


The terms $A_{i j}^{(m)}$ correspond to the initial layers $m=1-3$, with layers 1 and 3 being equivalent: $\varepsilon_{1, k}=\varepsilon_{3, k}$. We have

$$
\begin{array}{r}
A_{i j}^{(1)}(k, p)=A_{i j}^{(3)}(k, p)=b_{i}^{2}(k) b_{j}^{2}(p), A_{i j}^{(2)}(k, p)=g_{i}^{2}(k) g_{j}^{2}(p), \\
E_{i j}(k)=2 T_{k}\left[b_{i}^{2}(k) g_{j}^{2}(k)+b_{j}^{2}(k) g_{i}^{2}(k)\right],
\end{array}
$$

with $b_{1}(k)=1 / \sqrt{2}, b_{2}(k)=(1-a / d) u_{1} / 4, b_{3}(k)=(1+a / d) u_{2} / 4, g_{1}(k)=0, g_{2}(k)=t_{\perp, k} u_{1} / d, g_{3}(k)=t_{\perp, k} u_{2} / d$ and $u_{1}=\sqrt{2+(a+d)^{2} /\left(2 t_{\perp, k}\right)^{2}}, u_{2}=\sqrt{2+(a-d)^{2} /\left(2 t_{\perp, k}\right)^{2}}$.

For $\mathrm{M}=4$ layers $\xi_{(1,2), k}=\left(\varepsilon_{1, k}+\varepsilon_{2, k}+t_{\perp, k} \mp s_{1}\right) / 2, \xi_{(3,4), k}=\left(\varepsilon_{1, k}+\varepsilon_{2, k}-t_{\perp, k} \mp s_{2}\right) / 2$, with $d=\varepsilon_{1, k}-\varepsilon_{2, k}, s_{1,2}=$ $\sqrt{d^{2} \pm 2 t_{\perp, k} d+5 t_{\perp, k}^{2}}$. Here

$$
w_{i j}(k, p)=V_{1}(k, p)\left[A_{i j}^{(1)}(k, p)+A_{i j}^{(4)}(k, p)\right]+V_{2}(k, p)\left[A_{i j}^{(2)}(k, p)+A_{i j}^{(3)}(k, p)\right]+E_{i j}(k) \delta_{k p} .
$$

The terms $A_{i j}^{(m)}$ correspond to the initial layers 1-4, with layers 1 and 4 and also 2 and 3 being equivalent. Now

$$
\begin{array}{r}
A_{i j}^{(1)}(k, p)=A_{i j}^{(4)}(k, p)=b_{i}^{2}(k) b_{j}^{2}(p), A_{i j}^{(2)}(k, p)=A_{i j}^{(3)}(k, p)=g_{i}^{2}(k) g_{j}^{2}(p), \\
E_{i j}(k)=2 T_{k}\left[b_{i}^{2}(k) g_{j}^{2}(k)+b_{j}^{2}(k) g_{i}^{2}(k)+g_{i}^{2}(k) g_{j}^{2}(k)\right],
\end{array}
$$

with $b_{1}(k)=u_{1}\left(d+t_{\perp, k}-s_{1}\right) /\left(4 s_{1}\right), b_{2}(k)=u_{2}\left(d+t_{\perp, k}+s_{1}\right) /\left(4 s_{1}\right), b_{3}(k)=u_{3}\left(-d+t_{\perp, k}+s_{2}\right) /\left(4 s_{2}\right), b_{4}(k)=$ $u_{4}\left(d-t_{\perp, k}+s_{2}\right) /\left(4 s_{2}\right), g_{1}(k)=u_{1} t_{\perp, k} /\left(2 s_{1}\right), g_{2}(k)=u_{2} t_{\perp, k} /\left(2 s_{1}\right), g_{3}(k)=u_{3} t_{\perp, k} /\left(2 s_{2}\right), g_{4}(k)=u_{4} t_{\perp, k} /\left(2 s_{2}\right)$ and $u_{1}=\sqrt{2+\left(d+t_{\perp, k}+s_{1}\right)^{2} /\left(2 t_{\perp, k}^{2}\right)}, u_{2}=\sqrt{2+\left(d+t_{\perp, k}-s_{1}\right)^{2} /\left(2 t_{\perp, k}^{2}\right)}, u_{3}=\sqrt{2+\left(d-t_{\perp, k}+s_{2}\right)^{2} /\left(2 t_{\perp, k}^{2}\right)}, u_{4}=$ $\sqrt{2+\left(d-t_{\perp, k}-s_{2}\right)^{2} /\left(2 t_{\perp, k}^{2}\right)}$.

So far the formalism was quite generic. Specializing to Coulomb repulsion generated positive definite pairing potentials [12] for the cuprates, we consider the realistic non-separable form (and thus harder computationally)

$$
V_{i}(\vec{k}, \vec{p})=V_{i}(\vec{k}-\vec{p}), \quad V_{i}(\vec{q})=V_{o i} \sin ^{2}\left(q_{x} a\right) \sin ^{2}\left(q_{y} a\right), \quad V_{o i}>0
$$

which is peaked at (near) $\vec{Q}=( \pm \pi, \pm \pi)$ for $a=0.5(0.5<a<0.6)$. This type of potential is well known to generate a $d_{x^{2}-y^{2}}$-wave gap $[11,12]$.

Another relevant issue is the doping imbalance for $\mathrm{M}>2$ layers in the unit cell. Namely, NMR experiments show that the outer layers are overdoped with holes compared to the inner layers [17], in agreement with earlier theoretical estimates [5]. We account for this fact by typically considering $\mu_{\text {out }}=\mu-0.06 t, \mu_{\text {in }}=\mu+0.06 t$ for both $\mathrm{M}=3,4$, with $\mu_{\text {out }} / \mu_{\text {in }}$ referring to the chemical potentials of the original outer/inner layers and $\mu$ the chemical potential for the case $\mathrm{M}=1,2$. Assuming a screened electronic interaction, its strength is determined by the susceptibility $\chi(q, \omega)$ 12, 13]. It can be shown that $\chi(q, \omega=0)$ is a decreasing function of $\mu$ for the range of doping considered herein [12]. Therefore, taking $V_{o 1}=V_{o, \text { out }}>V_{o 2}=V_{o, \text { in }}$, reflects also the effect of $\mu_{\text {out }}<\mu_{\text {in }}$ for $\mathrm{M}=3,4$. In our model, this potential imbalance is more important than the sheer $\mu$ imbalance. Overall, $V_{i}(k, p)$ and $T_{k}$ (c.f. below) mostly determine the gaps $\Delta_{i, k}$ and hence $T_{c}$. This simply reflects the fact that both $V_{i}(k, p)$ and $T_{k}$ enter on an equal basis in the BCS gap equations - c.f. eqs. (9) and (11). The potential has a drastic influence on $T_{c}$, as is known from standard BCS-Eliashberg theory [11, 12].

We give a summary of a few calculations in the 3 figures and the table below, noting that they refer to $d_{x^{2}-y^{2}-\text { wave }}$ solutions of the gap equations above [18]. In all cases the energy scale is given by $t=1$, and $a=0.5$. Typically values $0.5<a<0.6$ yield higher $T_{c}$ 's, without a qualitative change of the results below.

In the table, an asterisk marks the highest $T_{c}$ in each case. $\mathrm{A}$ maximum $T_{c}$ for $\mathrm{M}=3$, without resorting to the potential imbalance $V_{o, \text { out }}>V_{o, \text { in }}\left(V_{1}>V_{2}\right.$ etc.), can be obtained for a small enough (in magnitude) pair hopping $T_{o}<0$, and this fact is facilitated by smaller values of $t_{o}$ - c.f. case A.1 and the figures. Otherwise, for $V_{o, o u t}=V_{o, \text { in }}$ and higher $T_{o}, \mathrm{M}=2$ yields the maximum $T_{c} . T_{c}$ depends weakly on the single particle hopping $t_{o}$. In ref. [8] it was shown that the bare value of $t_{o}$ is significantly reduced through the effect of interactions. The sign of $t_{o}$ is irrelevant, as can be seen from the gap equations. This is not the case for the sign of the pair-coherent term $T_{o}$ though (c.f. below). $T_{o}>0$, in general, does not reproduce the dome-type dependence of $T_{c}$ on $\mathrm{M}$ with a maximum for $\mathrm{M}=3$.

Given the strong dependence of $T_{c}$ on $T_{k}$ within our model, materials with $\mathrm{Y}$, Ba and Sr intercalants should have a much smaller $T_{k}$ than Ca-intercalated cuprates. Moreover, the in-plane pairing potential is expected to be weaker for the former materials for $\mathrm{M}>1$.

In all, we note that the experimental situation of the $T_{c}$ increasing for $\mathrm{M}=1-3$ (or 4 ) and then dropping, is only realized for rather small negative values of the pair tunneling amplitude $T_{o}$. E.g. for $T_{o}=-0.02 t \mathrm{M}=2$ yields 
A. $t^{\prime}=-0.35, t^{\prime \prime}=0, n=0.80, T_{o}=-0.002$

\begin{tabular}{|c|c|c|c|}
\hline 1. & $t_{o}=0.001$ & $V_{o 1}=V_{o 2}=4$ & $T_{c}(M=1-4)=(5.384,6.347, * \mathbf{6 . 4 2 4}, 6.241) 10^{-2}$ \\
\hline 2. & $t_{o}=0.01$ & $V_{o 1}=V_{o 2}=4$ & $T_{c}(M=1-4)=(5.384, * \mathbf{6 . 3 4 7}, 6.187,5.852) 10^{-2}$ \\
\hline 3. & $t_{o}=0.001$ & $V_{o 1}=V_{o 2}=3$ & $T_{c}(M=1-4)=(1.960, * \mathbf{2 . 9 4 3}, 2.806,2.611) 10^{-2}$ \\
\hline 3.a & $t_{o}=0.001$ & $V_{o 1}=3.2, V_{o 2}=3$ & $T_{c}(M=3,4)=(* \mathbf{3 . 4 0 3}, 3.240) 10^{-2}$ \\
\hline $3 . \mathrm{b}$ & $t_{o}=0.001$ & $V_{o 1}=3.5, V_{o 2}=3$ & $T_{c}(M=3,4)=(* \mathbf{4 . 4 0 6}$ \\
\hline \multicolumn{4}{|c|}{ B. $t^{\prime}=-0.35, t^{\prime \prime}=0, n=0.80, T_{o}=-0.02$} \\
\hline 1. & 01 & $V_{o 1}=4.5, V_{o 2}=4$ & $T_{c}(M=1-4)=(5.384, * \mathbf{1 4 . 6 8}$, \\
\hline \multicolumn{4}{|c|}{ C. $t^{\prime}=-0.3, t^{\prime \prime}=0.2, n=0.85, T_{o}=-0.002$} \\
\hline 1. & $t_{o}=0.001$ & $V_{o 1}=V_{o 2}=4$ & $T_{c}(M=1-4)=(1.215, * \mathbf{1 . 7 0 0}, 1.595,1.546) 10^{-2}$ \\
\hline 1.a. & $t_{o}=0.001$ & $V_{o 1}=4.5, V_{o 2}=4$ & $T_{c}(M=3,4)=(* \mathbf{2 . 2 6 4}, 2.216) 10^{-2}$ \\
\hline 2 . & $t_{o}=0.01$ & $V_{o 1}=V_{o 2}=4$ & $T_{c}(M=1-4)=(1.215, * \mathbf{1 . 6 3 2}, 1.382,0.9957) 10^{-2}$ \\
\hline a. & $t_{o}=0.01$ & $V_{o 1}=4.5, V_{o 2}=4$ & $T_{c}(M=3,4)=(* \mathbf{1 . 9 7 8}, 1.627) 10^{-2}$ \\
\hline
\end{tabular}

TABLE I: $T_{c}$ calculated as a function of the parameters shown.

the maximum $T_{c}$, as opposed to $\mathrm{M}=3$ for $T_{o}=-0.002 t$ - c.f. case D.3 below for a maximum for $\mathrm{M}=4$. The negative $T_{k}$ can be understood as follows. Within the frame of second order perturbation theory, we have the relation $T_{i l, k}=-2 t_{\perp, k}^{2} v_{i, k}^{(0)} u_{i, k}^{(0)} v_{l, k}^{(0)} u_{l, k}^{(0)} /\left(E_{i, k}^{(0)}+E_{l, k}^{(0)}\right)$, with the index (0) denoting the initial uncoupled layers, with $E_{i, k}^{(0)}=\sqrt{\left(\Delta_{i, k}^{(0)}\right)^{2}+\varepsilon_{i, k}^{2}}$. $T_{i l, k}<0$ follows from $E_{i, k}^{(0)}+E_{l, k}^{(0)}>0$.

We give the results of our calculations for five homologuous series of cuprates, which are similar to the cases above. We use a restricted set of parameters - $t=220-245 \mathrm{meV}, t^{\prime}=-0.35 t, t^{\prime \prime}=0, t_{o}=0.03 t, n=0.80$, which corresponds to optimal doping, $a=0.5, T_{o}=-0.0044 t-(-0.0018) t$, and $V_{o i}=2.7 t-4.2 t$ - to demonstrate the fitting capacity of our model. We obtain exact matches with the experimental $T_{c}$ values, given in degrees $\mathrm{K}$ and taken from ref. [1]. We also give the ratios $\mathrm{R}=\max \left\{\Delta_{i, k}(T=0)\right\} / T_{c}$, which turn out to be in the range 0.71-1. These values are too small compared to the actual experimental ones, pointing to the limitations of the BCS description for the cuprates. Along the same line, the $V_{o i}$ values are in the intermediate coupling regime, i.e. beyond the strict limit of applicability of the BCS framework. Note that the opposite potential imbalance $V_{1}<V_{2}$ would yield the same $T_{c}$ 's with somewhat higher values of $V_{2}$ for $\mathrm{M}=3,4$ than the $V_{1}$ values below. The values of $t$ and $V_{o 1}$ are adjusted so as to yield $T_{c}(\mathrm{M}=1)$. Then, $T_{o}$ is chosen so as to yield $T_{c}(\mathrm{M}=2)$. Subsequently, $V_{o 2}(M=3,4)=V_{o 1}(M=1)=V_{o 1}(M=2)$, and $V_{o 1}(M=3,4)$ are chosen so as to yield $T_{c}(\mathrm{M}=3,4)$.

D.1 $\mathrm{Bi}_{2} \mathrm{Sr}_{2} \mathrm{Ca}_{M-1} \mathrm{Cu}_{M} \mathrm{O}_{2 M+4+\delta}$ with $t=245 \mathrm{meV}$ and $T_{o}=-0.0044 t, V_{o 1}=V_{o 2}=2.7 t$. For $\mathrm{M}=3$ we take $V_{o 1}=3.5 t$, thus obtaining $T_{c}(\mathrm{M}=1-3)=36,90,110 \mathrm{~K}$ and $\mathrm{R}(\mathrm{M}=1-3)=0.769,0.713,0.867$.

D.2 $\mathrm{TlBa}_{2} \mathrm{Ca}_{M-1} \mathrm{Cu}_{M} \mathrm{O}_{2 M+3+\delta}$ with $t=229 \mathrm{meV}$ and $T_{o}=-0.0044 t, V_{o 1}=V_{o 2}=3 t$. For $\mathrm{M}=3$ we take $V_{o 1}=3.85 t$ and for $\mathrm{M}=4 V_{o 1}=4.16 t$, thus obtaining $T_{c}(\mathrm{M}=1-4)=52,107,133,127 \mathrm{~K}$ and $\mathrm{R}(\mathrm{M}=1-4)=0.765,0.781$, $0.973,1.034$.

D.3 $\mathrm{Tl}_{2} \mathrm{Ba}_{2} \mathrm{Ca}_{M-1} \mathrm{Cu}_{M} \mathrm{O}_{2 M+3+\delta}$ with $t=220 \mathrm{meV}$ and $T_{o}=-0.0024 t, V_{o 1}=V_{o 2}=3 t$. For $\mathrm{M}=3$ we take $V_{o 1}=3.67 t$ and for $\mathrm{M}=4 V_{o 1}=4.2 t$, thus obtaining $T_{c}(\mathrm{M}=1-4)=50,80,110,122 \mathrm{~K}$ and $\mathrm{R}(\mathrm{M}=1-4)=0.765,0.755$, $0.909,1.039$. This series has maximum $T_{c}$ for $\mathrm{M}=4$ - c.f. 2].

D.4 $\mathrm{Tl}_{2} \mathrm{Ba}_{2} \mathrm{Ca}_{M-1} \mathrm{Cu}_{M} \mathrm{O}_{2 M+4+\delta}$ with $t=221 \mathrm{meV}$ and $T_{o}=-0.0018 t, V_{o 1}=V_{o 2}=3.5 t$. For $\mathrm{M}=3$ we take $V_{o 1}=3.76 t$ and for $\mathrm{M}=4 V_{o 1}=3.83 t$, thus obtaining $T_{c}(\mathrm{M}=1-4)=90,115,125,116 \mathrm{~K}$ and $\mathrm{R}(\mathrm{M}=1-4)=0.795,0.845$, $0.934,0.976$.

D.5 $\mathrm{HgBa}_{2} \mathrm{Ca}_{M-1} \mathrm{Cu}_{M} \mathrm{O}_{2 M+2+\delta}$ with $t=238.5 \mathrm{meV}$ and $T_{o}=-0.0021 t, V_{o 1}=V_{o 2}=3.5 t$. For $\mathrm{M}=3$ we take $V_{o 1}=3.75 t$ and for $\mathrm{M}=4 V_{o 1}=3.89 t$, thus obtaining $T_{c}(\mathrm{M}=1-4)=97,127,135,129 \mathrm{~K}$ and $\mathrm{R}(\mathrm{M}=1-4)=0.765,0.851$, $0.932,0.979$.

In summary, higher $T_{c}$ in multilayer copper oxides arises from a relatively increased (in magnitude) pair interlayer coupling $T_{k}<0$, combined with a substantial strength of the repulsive Coulomb interaction between the electrons. It seems that all these ingredients are present in the Ca-intercalated materials. This picture should possibly be complemented by the $\mathrm{CuO}_{2}$ lattice symmetry effects [19]. The BCS approach, though of limited applicability in the cuprates, offers relevant insight. We give an interpretation of the negative $T_{k}$ in the BCS framework.

Stamatis Stamatiadis provided essential programming assistance. The author enjoyed discussions with Josheph Betouras, who also read critically the manuscript. Funding received from the Dept. of Materials Science of the 
University of Crete is acknowledged.

*E-mail address : kast@iesl.forth.gr, giwkast@gmail.com

[1] X.J. Chen and H.Q. Lin, Phys. Rev. B 69, 104518 (2004), and Phys. Rev. B 71, 109901(E) (2005).

[2] G. Burns, High-Temperature Superconductivity: An Introduction (Academic, Boston, 1992).

[3] A. J. Leggett, J. Phys. Chem. Solids 59, 1729 (1998).

[4] Z. Tesanovic, Phys. Rev. B 36, 2364 (1987).

[5] M. Di Stasio, K.A. Müller, and L. Pietronero, Phys. Rev. Lett. 64, 2827 (1990).

[6] S. Chakravarty, A. Sudbo, P.W. Anderson, and S. Strong, Science 261, 337 (1993).

[7] K. Byczuk and J. Spalek, Phys. Rev. B 53, R518 (1996).

[8] A. I. Liechtenstein, O. Gunnarsson, O. K. Andersen, and R. M. Martin, Phys. Rev. B 54, 12505 (1996).

[9] E. W. Carlson, S. A. Kivelson, V. J. Emery, and E. Manousakis Phys. Rev. Lett. 83, 612 (1999).

[10] S. Chakravarty, Hae-Young Kee, and K. Völker, Nature 428, 53 (2004).

[11] D.J. Scalapino, Phys. Repts. 250, 329 (1995).

[12] G. Kastrinakis, Physica C 340, 119 (2000).

[13] G. Kastrinakis, Phys. Rev. B. 71, 014520 (2005).

[14] P.G. de Gennes, Superconductivity of Metals and Alloys (Benjamin, New York, 1966).

[15] O.K. Andersen, A.I. Liechtenstein, O. Jepsen, and F. Paulsen, J. Phys. Chem. Solids 56, 1573 (1995).

[16] For $\mathrm{M}=3,4$

$$
U_{3}=\left(\begin{array}{ccc}
-b_{1}(k) & b_{2}(k) & b_{3}(k) \\
g_{1}(k) & -g_{2}(k) & g_{3}(k) \\
b_{1}(k) & b_{2}(k) & b_{3}(k)
\end{array}\right) \quad, \quad U_{4}=\left(\begin{array}{cccc}
b_{1}(k) & -b_{2}(k) & b_{3}(k) & b_{4}(k) \\
g_{1}(k) & -g_{2}(k) & g_{3}(k) & g_{4}(k) \\
-g_{1}(k) & g_{2}(k) & g_{3}(k) & g_{4}(k) \\
-b_{1}(k) & b_{2}(k) & b_{3}(k) & b_{4}(k)
\end{array}\right),
$$

with $b_{i}(k), g_{i}(k)$ as given after eqs. (13) and (15) respectively.

[17] H. Kotegawa, Y. Tokunaga, K. Ishida, G.-q. Zheng, Y. Kitaoka, K. Asayama, H. Kito, A. Iyo, H. Ihara, K. Tanaka, K. Tokiwa, and T. Watanabe, J. Phys. Chem. Solids 62, 171 (2001).

[18] We use a 64 by 64 discretization of the Brillouin zone, and we solve the full temperature dependent gap equations directly for the angles $\theta_{i, k}$.

[19] G. Kastrinakis, e-print arXiv:0901.0097.

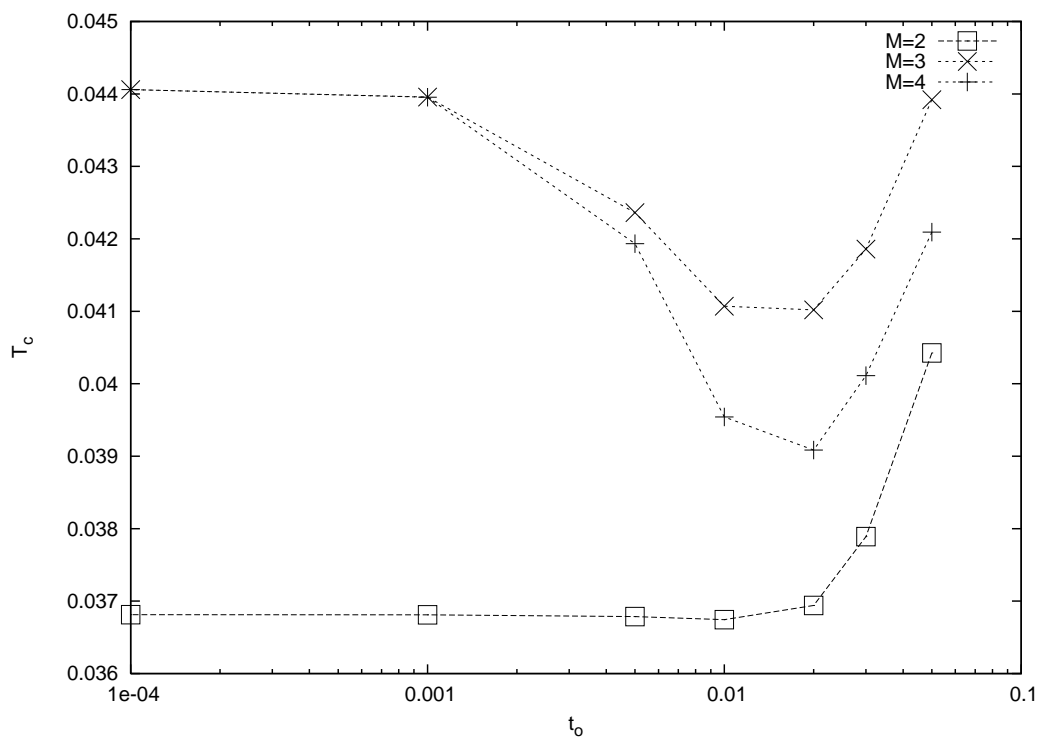

FIG. 1: $T_{c}$ (in units of $t$ ) as a function of interplane hopping for $n=0.85, t^{\prime}=-0.35, t^{\prime \prime}=0, V_{o 1}=V_{o 2}=4$. The inner/outer layer doping imbalance is taken into account for $\mathrm{M}=3,4$. Squares : $\mathrm{M}=2$, $\mathrm{x}$ 's : $\mathrm{M}=3$ and crosses : $\mathrm{M}=4$. $T_{c}$ versus $t_{o}$ is shown for $T_{o}=0$. All lines are guides to the eye. 


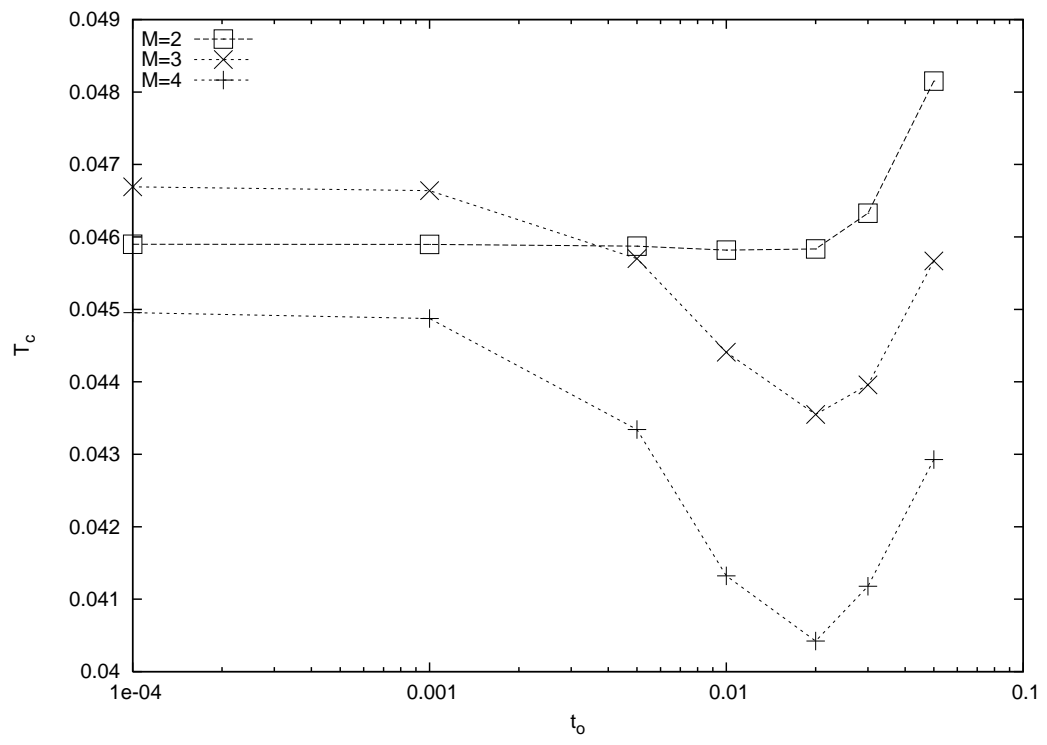

FIG. 2: Same as in fig. 1. $T_{c}$ versus $t_{o}$ for $T_{o}=-0.002$.

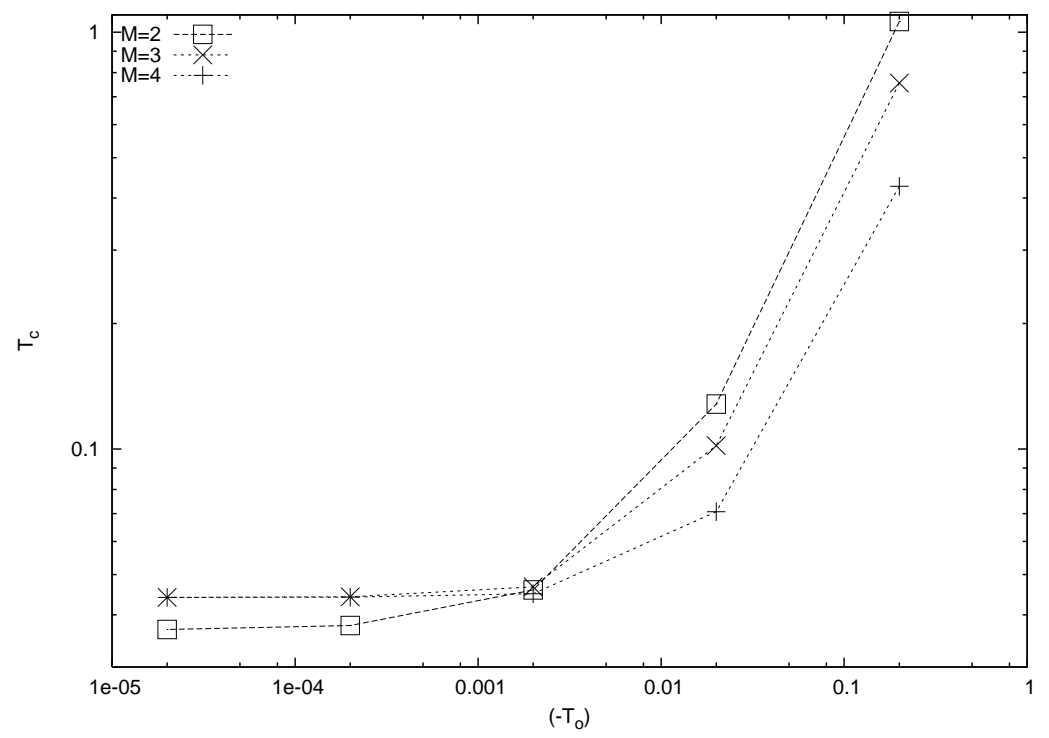

FIG. 3: Same as in fig. 1. $T_{c}$ versus $\left(-T_{o}\right)$ for $t_{o}=10^{-7}$. Notice the log scale for $T_{c}$. 